\title{
COMPARING USEFULNESS OF HUMIDIFIED HIGH-FLOW NASAL CANNULA (HHFNC) AND NASAL CONTINUOUS POSITIVE AIRWAY PRESSURE (NCPAP) FOR NEONATAL RESPIRATORY DISEASES IN PRETERM INFANTS
}

\author{
B.M. Choi ${ }^{1,2}$, E.H. Lee ${ }^{1}$, K.H. Park ${ }^{1}$, B.H. Chung ${ }^{1}$, H.J. Park ${ }^{2}$, Y.O. Choi ${ }^{2}$, Y.S. Hong ${ }^{1}$ \\ ${ }^{1}$ Departement of Pediatrics, Korea University College of Medicine, Seoul, ${ }^{2}$ Departement of Pediatrics, \\ Korea University Ansan Hospital, Ansan-Si, Republic of Korea
}

Background and aim: HHFNC is a widely used respiratory supportive mode for premature infants because of its relatively ease use and good compliance of infants as an alternative of nCPAP in many NICU. The aim of this study is to assess the clinical effectiveness and safety of a new HHFNC device (RT329 Infant Oxygen Delivery System) compared to nCPAP for neonatal respiratory diseases in preterm infants.

Methods: Preterm infants who need respiratory support due to neonatal respiratory disease were supplied by HHFNC $(n=35)$ or nCPAP $(n=35)$. Primary outcomes included the rate of weaning and the rate of failure defined as intubation with mechanical ventilator care within 72 hour. Other outcomes included the duration of these respiratory supports, the improvement of vital signs (heart rate and respiratory rate), $\mathrm{FiO}_{2}$ needed for respiratory stabilization and the rate of complications (localized infection and necrosis, air leak syndrome, etc) after HHFNC or nCPAP.

Results: There were no significant differences in demographics of patients. $65.7 \%(23 / 35)$ infants in HHFNC group weaned within 72 hours as compared with $74.3 \%(26 / 35)$ infants in nCPAP group $(P=0.438)$. $20 \%(7 / 35)$ infants in HHFNC group were intubated compared with $17.1 \%(6 / 35)$ infants in nCPAP group $(P=0.603)$. There were no significant differences in the duration of these respiratory supports and other outcomes.

Conclusions: This study indicates that HHFNC may be used as an effective and safe alternative of nNCPAP in the preterm infants. But well-designed randomized study is necessary for clinical application of HHFNC. 